\title{
PARA UNA NUEVA FORMA DE EJERCER LA COMUNICACIÓN: RUTAS Y PERSPECTIVAS DEL COMUNICADOR ACTUAL
}

\section{NEW WAY TO PRACTISE COMMUNICATION: ROUTES AND PERSPECTIVES OF THE OF THE PRESENT-DAY COMMUNICATOR}

\section{MARÍA ISABEL IMBAQUINGO P. ${ }^{1}$ CARlos Aulestia P. ${ }^{2}$}

Recibido: 31 de enero de 2017 Aceptado: 6 de marzo de 2017

\footnotetext{
${ }^{1}$ Pontificia Universidad Católica del Ecuador, Facultad de Comunicación, Lingüística y Literatura, Escuela de Comunicación, Quito, Ecuador, (miimbaquingo@puce.edu.ec).

${ }^{2}$ Pontificia Universidad Católica del Ecuador, Facultad de Comunicación, Lingüística y Literatura, Escuela de Comunicación, Quito, Ecuador, (chaulestia@puce.edu.ec).
} 



\section{PARA UNA NUEVA FORMA DE EJERCER LA COMUNICACIÓN: RUTAS Y PERSPECTIVAS DEL COMUNICADOR ACTUAL}

\section{A NEW WAY TO PRACTISE COMMUNICATION: ROUTES AND PERSPECTIVES OF THE OF THE PRESENT-DAY COMMUNICATOR}

María Isabel Imbaquingo P., Carlos Aulestia P.

PALABRAS CLAVE: comunicador, Mass Media, nuevas tecnologías, modernidad líquida, mediación social, cambio social

KEY WORDS: communicator; "Mass" media; new technologies; liquid modernity; social mediation; social change

\section{RESUMEN}

Este artículo examina las condiciones académicas actuales para la generación de un nuevo perfil de comunicador, cuya preparación profesional le permita internarse de una forma distinta y activa en el ámbito sociocultural nacional y regional. Este nuevo campo de intervención del comunicador se asienta en la superación de paradigmas que han perdido vigencia en la práctica concreta de la profesión, considera el papel de la tecnología únicamente como una herramienta que facilita ciertos procesos comunicativos y enfatiza la necesidad de una sólida formación teórica que dé profundidad a la actividad del comunicador, 
tanto como un analista de los fenómenos socioculturales cuanto como un gestor de cambios y transformaciones estructurales en el entorno cultural.

Para lograr tales objetivos, se propone la revisión y la actualización del concepto de mediación social, propuesto por Manuel Martín Serrano, como un principio para la comprensión de las contradicciones inherentes a una sociedad cada vez más dependiente de la hegemonía tecnológica y otros modelos de mundo en los que, paradójicamente, no se ven cambios estructurales profundos que permitan romper con la estructura actual del sistema social, sus desequilibrios, desbalances y formas de ejercer poder.

Se revisa además, como un antecedente de la comunicación para el cambio social, las propuestas de Jesús Marín Barbero sobre una comunicación social participativa en contextos sociales como el Latinoamericano, entendido no desde teorías externas sino desde los propios procesos comunicativos generados en la convivencia real de la población y sus prácticas culturales.

\section{ABSTRACT}

This article examines the current academic conditions required of a new generation of Communicators, whose professional training allows them to commit in a different and active way to all national and regional socio-cultural fields. This new field of Communicator's involvement is based on overcoming paradigms that have lost relevance in the specific practice within the profession. It considers the role of technology only as a tool that facilitates certain communicative processes, and emphasizes the need of a solid theoretical training that gives depth to the role of the Communicator as an analyst of the socio-cultural phenomena and an agent for changes and structural transformations in the cultural environment.

To achieve such objectives, the review and updating of the concept of social mediation (proposed by Manuel Martín Serrano) is recommended as a principle for the understanding of contradictions inherent in a society increasingly dependent on technological hegemony which, paradoxically, does not show any deep structural changes that allow to break with the current structure of the social system, its imbalances, instabilities and ways of exerting power.

As a precedent of communication for social change, there is a review of Jesus Marin Barbero's proposals about 
participative communication in social contexts (such as Latin American), understood not from external theories but from their own social procedures generated by the actual coexistence of the population and their cultural practices.

\section{ANTECEDENTES Y CONTEXTO}

Es indiscutible que, en los últimos años, la formación profesional en comunicación ha sufrido un cambio importante en sus alcances sociales, campos de acción y fundamentos epistemológicos. Como consecuencia de ello, las universidades que se ocupan de formar comunicadores, así como los profesionales que se dedican a estas tareas, se han visto en la necesidad de repensar y replantear numerosos principios de la profesión, algunos más prácticos, otros más conceptuales, con la finalidad de garantizar la viabilidad de la acción del comunicador dentro de la dinámica social.

El viejo paradigma del comunicador como periodista o como vocero de entidades corporativas públicas y privadas, ha quedado, a la luz de las nuevas formas de organización de los medios y las nuevas maneras de relacionarse de las empresas y la sociedad, definitivamente caduco y desactualizado.

El papel del periodista tradicional, incrustado en la dinámica habitual de los medios: buscar la noticia, redactarla, escribirla, editarla, difundirla, es cada vez menos demandado en las organi- zaciones mediáticas. Se requiere en la actualidad otro tipo de profesional que cumpla con las labores periodísticas de una forma más funcional y eficiente mediante el uso de las nuevas tecnologías de la comunicación y los dispositivos electrónicos. De igual forma, el comunicador empresarial que se ocupa de organizar eventos, establecer contactos con medios y, en el mejor de los casos, producir algún producto de comunicación interna o externa, es, en la actualidad, una especie en extinción dentro de los ámbitos organizacionales.

Así pues, el objetivo de este artículo será señalar y argumentar la viabilidad actual y vigencia de nuevas tendencias en la formación del comunicador que superan la dicotomía 'comunicador organizacional/periodista', las cuales, a partir de una reflexión epistemológica compartida entre la academia o otras muchas organizaciones de la sociedad civil, trazan un perfil novedoso, actual y máximamente funcional en las dinámicas socioculturales actuales; en estas, la profesión encuentra nuevos campos de ejercicio de sus destrezas y 
conocimientos, mayores posibilidades de empleabilidad, y una muy variada y amplia oportunidad de contribuir en los procesos sociales más actuales y decisivos mediante un ejercicio profesional que, apuntalado por las nuevas tecnolo- gías y por el manejo de las mediaciones sociales como formas comprensión de las relaciones entre los actores culturales, genera escenarios comunicativos emergentes y perentorios en las relaciones del mundo actual.

\section{DE LOS “MASS" MEDIA A LA RED GLOBAL: LA PROBLEMÁTICA DEL COMUNICADOR}

¿Cómo desligar al comunicador de la dinámica de los mass media y los roles tradicionales de la empresa e insertarlo de una manera influyente y efectiva en el escenario comunitario, de manera que su participación como agente social y profesional esté aún vigente y muestre posibilidades de crecimiento y expansión? Esta es la pregunta que las instituciones académicas, universidades, institutos de formación profesional, deben formularse actualmente para definir el problema de la reflexión epistemológica y práctica de los programas de formación profesional del comunicador tanto a nivel de pregrado como de posgrado.

La problemática que surge en torno a esta pregunta es acaso una de las más complicadas y delicadas en la historia de la reflexión teórica sobre el periodismo y la comunicación social, dada una coyuntura mundial en la que confluyen numerosos e inéditos factores como la irrupción de las redes sociales, y su secuela natural, la crisis del periodismo tradicional en sus formatos habituales (prensa escrita, radio y televisión). En el contexto de los años 90, sobre todo, y aun en los años recientes, surgieron posturas que vaticinaron el final de los medios de comunicación de masas, bajo el argumento que su modelo de producción se había acabado:

Los periódicos han dejado de ser atractivos para el joven consumidor, particularmente en países desarrollados, donde la sociedad del conocimiento ha impulsado modelos educativos con base en nuevas tecnologías que han trastocado la forma en allegarse de información. (Rodríguez, 2013)

En efecto, la crisis del periodismo impreso obligó a los medios tradicionales a replantear sus formas de producción y la forma de transmisión de sus 
contenidos. Hubo casos en que grandes medios masivos anunciaron el fin de sus ediciones impresas para promocionar su sitios web. El periodista Matías Martínez Molina sostiene que

No solo la prensa, sino también todos los medios de comunicación -televisión abierta y de pago, radio, revistas, libros etc. - están en el centro de una revolución provocada por Internet y la banda ancha. Nadie sabe para donde van esos medios ni cuales serán las consecuencias de esa revolución. Pero es evidente que todos ellos deberán cambiar profundamente sus estructuras para adaptarse a las nuevas tecnologías. Uno de los nuevos fenómenos cada vez más evidente es la extraordinaria fragmentación de los mercados de comunicación. (2010, p. 148).

Hemos visto, al pasar estos años, que el apocalipsis de los medios no se ha producido; lo que sí es real e imposible de desconocer es la importancia que han alcanzado las ediciones vía web y la producción digital en comparación con los formatos tradicionales. Las ventajas son fáciles de deducir: mayor celeridad en la producción y distribución del mensaje, mayor participación del receptor, que se ha convertido en un coautor de la información, acceso notablemente más amplio al público. Lo mismo se puede decir de la radio y la televisión, cuyas dinámicas de trabajo no son las mismas desde el aparecimiento y evolución de las nuevas tecnologías.

\section{NUEVAS TECNOLOGÍAS, OPORTUNIDADES Y RIESGOS}

Las nuevas tecnologías, las redes sociales, los sistemas de "microblogging", anunciados como una nueva era de las relaciones sociales de comunicación, más democrática y participativa (las ponderadas 'comunidades en red'), se enfrentan en la actualidad a grandes detractores, como el recientemente fallecido sociólogo polaco Zigmun Baumann, cuya teoría de la sociedad actual concebida como una 'modernidad líquida' en la que nada tiene un valor estable se debe en gran medida la sobreabundancia de información hueca y superficial que estupidiza a los grupos sociales, les arrebata la memoria y la capacidad de retener valores y contenidos, confiándoselos a las tecnologías computarizadas. Al respecto, Jacobo Bañuelos se refiere al deterioro simbólico que implica una nueva tecnología, la fotografía digital, en estos términos: 
La hiper-abundancia (sic) cede paso a la acumulación y al olvido, quizás también a la amnesia, parece solo interesarnos recordar un presente continuo, parece ser que estamos cada vez más instalados en una especie de memoria del tiempo real, si es que esto pueda existir. Algo así como ver para olvidar. La facilidad de la imagen parece favorecer más a la cantidad de a lo que conocíamos como "calidad fotográfica", y parece desvanecer a la memoria, hacerla volátil, aunque estará por verse, porque hay dos grandes potencias en la naturaleza digital, una es la socialización y la otra es justamente la memoria. Quizás no recordemos nosotros pero sí las máquinas. (Bañuelos, 2013, p. 12)

En la misma línea, el comunicólogo Luis Torres Nabel, en su análisis del empleo de la red social Twitter en México, concluye su trabajo con las siguientes reflexiones:

En suma (...), la metodología de investigación sobre el fenómeno de las redes sociales en Internet es un campo que inicialmente está poblado por muchas indefiniciones conceptuales e instrumentales, teniendo así como mencionan Drezner \& Farrell (2008) un fenómeno aún bastante ambiguo y términos baumanianos bastante "líquido". (Torres Nabel, 2013, p. 168)

Este escepticismo actual contrasta con el entusiasmo que despertó pocos años atrás, en la década del 2000, el desarrollo incontenible de Internet, especialmente en el campo del periodismo, en el que estudiosos, sobre todo de la Universidad de Navarra, como Ramón Salaverría y Javier Díaz Noci, para hablar estrictamente de teóricos del ámbito hispanoamericano, reconocieron en las nuevas tecnologías oportunidades de crecimiento y democratización de la información, absolutamente jerárquica en las estructuras mediáticas tradicionales.

Los principios básicos de las nuevas tecnologías: la interactividad, la multimedialidad y, sobre todo, la hipertextualidad, ofrecen, para los entusiastas de estos adelantos técnicos, una nueva forma de distribución y transmisión de la información que menoscaba la jerarquía absoluta de los medios como únicos emisores de los mensajes que se transmiten en la sociedad.

Además, en los ambientes corporativos, el comunicador, en el contexto latinoamericano, fue, hasta los años 90, un funcionario de rango mediano cuyas funciones se limitaban a las relaciones públicas, el contacto de la organización con medios de difusión masiva y la organización de eventos. Este limitado perfil se expandió, también con el auge de las 
nuevas tecnologías. Teóricos organizacionales describen cómo las herramientas web crearon numerosas y amplias oportunidades para el desarrollo de la comunicación interna. En el ámbito externo, las tecnologías de la información posibilitaron que las grandes organizaciones generaran estrategias comunicativas a escala mundial, permitieron el desarrollo del e-learning y expandieron su imagen por medio de una visibilización global que actualmente casi llega a la ubicuidad. Por su parte, las pequeñas y medianas empresas se dieron a conocer a un público mucho más extenso y variado por medio de páginas web, "blogs" y diversas herramientas y aplicaciones. El comunicador organizacional debió convertirse, en este contexto, en un gestor de comunidades sociales cuya principal herramienta de trabajo es la web.

Así pues, la función social del comunicador, en pocos años, sufrió una serie de modificaciones como producto de la presión que las nuevas tecnologías impusieron a los profesionales de la comunicación. De pronto, cualquier reportero de un medio escrito debía convertirse en un fotógrafo que produce además video y audio, pues cuenta con los elementos tecnológicos para hacerlo (cualquier dispositivo inteligente). En radio y televisión, en cambio, los periodistas se vieron en la necesidad de empezar a escribir, pues los medios audiovisuales, en sus versiones web, se convierten en complejos productos multimedia que incluyen "blogs", bitácoras y foros.

Como parte de esta transformación de la función del comunicador, se han emitido propuestas de nuevas formas de periodismo de carácter marginal. Ciertas exitosas experiencias de cobertura mediante la red social Twitter, así como reportes espontáneos transmitidos, vía imagen, texto o video, por individuos que no son periodistas pero sí testigos privilegiados de acontecimientos noticiosos, generaron propuestas teóricas que resultaron débiles y pasajeras, como el 'periodismo ciudadano' o el 'periodismo móvil'.

\section{LA COMUNICACIÓN: MÁS ALLÁ DE LOS MEDIOS Y LA CORPORACIÓN. INVESTIGACIÓN Y MEDIACIÓN SOCIAL}

Una vez expuesto este diagnóstico de la etapa histórica más reciente de la comunicación, en relación con las funciones y el campo de intervención social del comunicador, corresponde, como el punto nuclear de este artículo, 
reflexionar sobre nuevas propuestas de un perfil de profesional que corresponde a la actualidad social ecuatoriana y latinoamericana, el cual, en cierto sentido, recupera los hallazgos y experiencias de las prácticas actuales en los ámbitos periodísticos y organizacionales, pero los profundiza, rebasa, desarrolla y complementa, para generar un conjunto de destrezas que, además de permitir al comunicador desempeñarse con éxito en los ámbitos señalados anteriormente, le capaciten para constituirse en un mediador y un gestor social cuya función primordial sea elaborar y construir discursos que ayuden a establecer entendimientos entre los distintos actores sociales, generar estrategias participativas de comunicación que permitan gestionar conflictos, diagnosticar situaciones de incomunicación, o reconocer códigos comunitarios marginales que constituyan elementos transversales de procesos de investigación científica a través de la mediación cultural imprescindible en el contacto entre el investigador y los actores sociales.

La investigadora Paula Morabes (2013), quien trabaja procesos de comunicación emergentes, investigación de la comunicación y educación, plantea, en su trabajo La fundamentación teórica problemáticas emergentes y líneas de investigación en comunicación/ educación: algunas reflexiones iniciales ante la imple- mentación de políticas públicas de inclusión educativas en Argentina,

(así) trabajar lo comunicacional, como un modo de construcción de objeto de estudios en las investigaciones, es asumirlo como una "perspectiva" o "mirada" para favorecer el proceso de desnaturalización del objeto. Buscando establecer un movimiento para pensar -siguiendo a Bourdieu- a lo "concreto" en términos de cada vez mayores niveles de abstracción y complejidad. Digamos, parafraseando al mismo Bourdieu, en su crítica a la sociología espontánea, salir de la "comunicación espontánea" (Morabes, 1913).

Propone la autora que la comunicación es, más que una práctica destinada a difundir información referencial en la sociedad, o una metodología para distribuir mensajes y discursos ya instituidos, una forma de aproximarse a fenómenos y objetos comunicativos que, como dispositivos discursivos cargados de significados impuestos por determinaciones y normas previamente establecidas, exigen una 'desnaturalización', en el sentido que estos discursos deben ser nuevamente examinados como mensajes neutrales despojados de sentidos deterministas y destinados a resignificarse, en los procesos de investigación e inter- 
pretación, según los códigos propios de los actores comunicativos.

La mirada del comunicador es, pues, una clave para descifrar diversos discursos, y su intervención consistirá en internarse en los procesos en los que participan sectores sociales que, en sus vínculos, necesitan optimizar y llegar a conocer las maneras en que deben erigirse como emisores de un mensaje, las estrategias necesarias para construir un mensaje claro y efectivo, las formas de difundirlo y ponerlo en circulación en diversos ámbitos, las técnicas y tecnologías de difusión más adecuadas para cumplir con estos objetivos, y la manera de leer y comprender los resultados obtenidos en estos procesos mediante el análisis y la comprensión de la retroalimentación recibida de los otros actores sociales. La tarea del comunicador es, precisamente, investigar, reflexionar, generar estrategias y gestionar el cumplimiento de estas etapas de los procesos comunicativos que se entablan en las relaciones sociales.

Lo dicho implica que el paradigma formativo del comunicador ha de cambiar radicalmente. La formación teórica debe ser lo más consecuentemente posible con las prácticas descritas como la nueva función social del comunicador. En este sentido, la abundancia de contenidos relativos a teorías comunicativas externas al medio social en la que el profesional ejercerá sus funciones resulta meramente informativa y hasta accesoria. El perfil del comunicador que se propone implica un profundo conocimiento de la actualidad de su entorno sociocultural y ambiental, así como el manejo fluido de contenidos contextuales, históricos, geográficos, antropológicos y deontológicos. Esto le proporcionará una base teórica cuyo dominio será fundamental para cumplir adecuadamente su función en una situación comunicativa.

Revisemos una cita de Raquel Aparicio, de la Universidad de Guadalajara, quien, en su trabajo sobre comunicación ambiental, propone, a manera de base conceptual, lo siguiente:

El primer paso fue conocer el significado que otorgan a la comunicación algunos autores hispanoamericanos contemporáneos, cuyas propuestas son más aproximadas al encuentro social que a las teorías "tradicionales" relativas a los medios y la comunicación masiva. Encontramos que, para Martín-Barbero (2008), la comunicación representa una dimensión constitutiva de la vida social; Portal (2008) la describe como una entidad conformadora de procesos culturales tendientes a la formación y consolidación de la subjetividad individual y colectiva; Galindo (1987) 
y Martín Serrano (1986/2009) la explican como una entidad que resulta central en la reproducción social y que es clave en las perspectivas de cambio y de transformación social; para Massoni (2008), se trata de un fenómeno cognitivo sociocultural, en tanto que León (2009) la concibe como un "proceso, práctica, espacio y columna vertebral de los significados del mundo y de las acciones e interacciones de los sujetos sociales" (Aparicio, 2016 p. 2016).

Esta cita nos interesa porque implica una idea que resulta fundamental para proponer un cambio de función innovador y consecuente con el contexto social en el que nuestras escuelas de Comunicación se desarrollan: por su estrecha relación con los ámbitos sociales más cercanos y propios, la formación del comunicador debe partir de teorizar sus problemas y sus fenómenos comunicativos de una manera exacta y bien delimitada; no de otra manera se puede reconocer cuáles son los objetivos de la acción del comunicador ni el valor social de su labor.

Aparicio menciona a varios teóricos latinoamericanos, unos más recientes y otros verdaderos clásicos, cuyo pensamiento necesariamente deberá revisar en su formación académica todo comunicador en ciernes.
Es, por tanto, esencial el dominio de los conceptos, las definiciones y los límites de las teorías de la comunicación que más se acercan y adaptan a los fenómenos simbólicos y discursivos de nuestro entorno tanto sociocultural como ambiental. Hay que comprender la lógica de estos conceptos, su articulación dentro de las propuestas teóricas que les dan sentido.

Acaso por la novedad que implicó el aparecimiento y auge de las nuevas tecnologías, las reflexiones sobre las funciones del comunicador sufrieron un aplazamiento bastante perjudicial que opacó el debate sobre las necesidades de cambio que la epistemología latinoamericana de la comunicación había ya iniciado, desde los trabajos de Jesús Martín Barbero, Manuel Martín Serrano y otros autores, cuyo concepto fundamental, las mediaciones sociales, es un punto de partida o un núcleo de pensamiento comunicacional que no ha perdido vigencia.

En este sentido, es interesante constatar que ya hace más de una década, en el año 2003, la reflexión sobre la necesidad de replantear el papel social del los comunicadores se había constituido en un tema de primera línea en el campo académico. El profesor Manuel Ortiz Marín, de la Universidad de Baja California, planteaba en ese año la urgente necesidad de repensar y volver a centrar 
en su contexto al comunicador social latinoamericano. Ortiz sostiene que:

... un espacio más cercano a lo social lo representa la participación del comunicador en las organizaciones de carácter ciudadano. Es el comunicador, desde la perspectiva de la construcción de la comunicación ciudadana, para la atención de problemas como: en la educación del trabajador (sic), al interior de los sindicatos; del ciudadano, en la defensa de sus derechos humanos; en lo cultural con los indígenas; en la perspectiva de género; en la comunicación popular, al interior de pequeñas organizaciones de barrio, y entre otros espacios ciudadanos igual de significativo. (Ortiz, 2003, p.35)

\section{EL ESPACIO LOCAL COMO LOCUS DE REFLEXIÓN TEÓRICA}

La perspectiva teórica que proponemos como base de la práctica comunicativa en la actualidad, radica fundamentalmente en un pensamiento que responda a las necesidades de las problemáticas comunicativas cercanas, propias, locales, comunitarias. Los problemas sociales que debe atender el comunicador, antes que nada, son los que se remiten a su ámbito cultural. En el caso del Ecuador y, en general, de varios sectores de la Región, en países como México, Perú, Colombia o Bolivia, este ámbito es especialmente fecundo: la hibridación cultural, en términos de Néstor García Canclini, produce fenómenos simbólicos y comunicativos cuya complejidad necesita ser despejada mediante análisis pertinentes y bien delimitados. No de otra manera podremos en- tender la complejidad de las abigarradas manifestaciones culturales latinoamericanas, muchas veces despreciadas como simples muestras de folclore, alienación o falta de educación y buen gusto.

El campo de las manifestaciones culturales populares está enteramente por registrarse y estudiarse. Desde una óptica pertinente teóricamente, como la hibridación cultural de García Canclini, es posible comprender y dar valor a las expresiones populares, cuya influencia social es mayor de lo que se puede pensar. El nuevo comunicador, como un analista de los procesos históricos de consolidación de las manifestaciones culturales, es capaz de comprender y explicar estos fenómenos en apariencia tan poco legibles y contradictorios. Ortiz Marín (2003) sostiene que: 
Otro campo de práctica de lo comunicacional está en lo popular. Es decir, en torno a las formas de organización de los sectores populares y de resolución de sus necesidades. Lo popular es el espacio mediante el cual dichas clases se organizan, manifiestan y reproducen lo popular a fin de resolver sus necesidades cultura- les o sociales. Lo anterior se explica, a partir de que el Estado reconoce que no puede ofrecer bienes y servicios suficientes para la satisfacción social y cultural de las clases, y tiene que aceptar de estas, sus propias manifestaciones culturales y sus modelos de organizaciones sociales. (p.36)

\section{EL ANÁLISIS DE LAS MEDIACIONES SOCIALES COMO DESMONTAJE DE LOS PROCESOS COMUNICATIVOS}

El concepto de mediación social parece retomar vigencia nuevamente en el contexto Latinoamericano, frente a los procesos sociales y económicos que vive en la actualidad la Región. En el campo teórico, las mediaciones sociales explican el proceso contradictorio por medio del cual la innovación tecnológica se opone a la dinámica cultural dada. De esta contradicción pueden surgir dos posibilidades: o bien la sociedad se ajusta a las imposiciones de la innovación tecnológica y busca adaptarse a ellas, para seguir reproduciendo el modelo; o bien el sistema social se rebela y rompe la relación para tratar de generar otro tipo de sociedad.

Podemos ejemplificar este fenómeno examinando lo que ocurre con las redes sociales, la televisión por cable y otros adelantos tecnológicos en nuestro medio: grandes segmentos de la población que carecen, por ejemplo, de servicios básicos o acceso a la educación media cuentan, sin embargo, con televisión satelital o teléfonos inteligentes.

En el contexto nacional y regional actual, tal situación implica una asimilación y una adaptación pasiva a las normas del sistema, un fenómeno que corresponde a lo que Martín Serrano describe como "asimilar tecnologías revolucionarias, sin necesidad de proceder a cambios estructurales profundos" (Martín Serrano, 1977, p. 39).

Las mediaciones sociales operan, según Martín Serrano, como mecanismos regulatorios que hacen posibles estas contradicciones, y se vuelven institucionales y actúan como principios cognitivos en los individuos, quienes, por estos mismos principios, no son ca- 
paces de advertir la disonancia y menos aún alcanzar sobre ella una capacidad crítica, lo cual refuerza el sistema.

La sociedad genera así una suerte de 'orden' establecido que se expresa por las mediaciones, que representan el poder real que rige la sociedad, cuya lógica es el mantenimiento del sistema. Las mediaciones son, pues, modelos para interpretar de determinada manera la realidad.

Estos modelos excluyen en el individuo la capacidad cognitiva de comprender cómo funciona el sistema en términos reales, y cuál es su lógica y su dinámica de funcionamiento. Así, se borra la posibilidad de advertir la contradicción.

Es posible construir un modelo lógico de una realidad contradictoria, poniendo en orden a nivel formal el desorden existente a nivel real, y (...) la actuación sobre la realidad sirviéndose de dicho modelo ideológico no ofrece ninguna dificultad especial. (...) La incoherencia en la organización social puede ser representada y estabilizada a nivel de un modelo formalmente racional. (Martín Serrano, 1977, p. 38)

El nuevo comunicador ha de partir de la comprensión teórica de qué es y cómo funcionan las mediaciones socia- les y cuál es su rol dentro de los sistemas culturales, ha de ser capaz de identificar las contradicciones entre la innovación tecnológica, el poder y la dinámica cultural concreta. Para lograr este objetivo, Manuel Martín Serrano considera que es indispensable el análisis lógico de la mediación por medio del reconocimiento de los códigos por medio de los cuales esta se hace inteligible. Estos códigos están destinados a construir relatos, que funcionan como grandes discursos orientadores de las instituciones que ejercen el control social. Así, en una cultura totalitaria y machista, por ejemplo, las mediaciones que institucionalizan estos comportamientos (las reglas de vestimenta, por ejemplo; los relatos cinematográficos, por ejemplo) pueden ser analizadas a partir de los códigos que las componen y les dan sentido en forma de relatos que actúan como representaciones totalizantes y arquetípicas de la realidad.

No son, pues, las conductas comunicativas las que deben someterse a análisis, sino las normas que regulan esas conductas y prácticas. La comprensión de la lógica de estas reglas, con sus restricciones y permisiones, dará paso al nivel ideológico de la mediación.

Así, el comunicador es un observador y un analista de los procesos socioculturales que comprende que estos no constituyen manifestaciones azaro- 
sas o casuales de la convivencia humana. Entiende que las relaciones a escala social se configuran como un conjunto de mensajes articulados por un agente mediador que ofrece un 'modelo de realidad', el cual obedece a la primacía de fuerzas sociales que ejercen poder sobre otras que se asimilan, para lograr así mantener el sistema. Este poder se expresa mediante relatos o articulaciones narrativas que conforman las reglas de asimilación y aceptación que plantean y sostienen el sistema.

El comunicador, desde esta perspectiva teórico-metodológica, adquiere una función social verdaderamente preponderante en la sociedad actual, pues su actividad constituye un ejercicio fundamental para reconocer, diagnosticar y proponer soluciones a conflictos socioculturales generados en las desiguales dinámicas de poder que se transfieren a la vida social por la vía de los agentes mediadores y las mediaciones. Es su tarea desmontar estos 'modelos de mundo' y despojarlos de los significados y funciones que les da el poder, para conseguir generar, desde una nueva mirada que 'desnaturaliza' el objeto, es decir, lo observa excluyendo las características que el poder artificialmente le ha ido atribuyendo según su lógica y su ideología. De esta forma, el comunicador po- drá desnaturalizar, releer y reinterpretar los fenómenos comunicativos tal cual se presentan en un momento y un contexto espacial determinado que le permitan investigarlos y conocerlos realmente y a profundidad.

Estas propuestas son compatibles teórica y prácticamente con las de Jesús Martín Barbero, que sostiene que la investigación de la comunicación se ubica en las esfera de las ciencias sociales mediante la generación de espacios comunicativos en los que las expresiones populares sean visibles, analizables y generen un impacto social necesario en contraposición con las representaciones de los medios de comunicación y las estructuras de poder. Para Quinche Ortiz, Martín Barbero propone

invertir lo que hasta entonces se había considerado: en vez de ver a la comunicación como proceso de dominación, ver a la dominación como proceso de comunicación, es decir, como proceso social y campo de batalla cultural, sostenido en pensadores como Hegel y Martín Serrano, quienes le van a permitir evidenciar las complicidades y seducciones que se establecen en las relaciones entre dominador y dominado. (Ortiz Crespo, 1998, p.63) 


\section{COMUNICACIÓN PARA EL CAMBIO SOCIAL}

En este contexto teórico, en la actualidad el reto del comunicador es rescatar y preservar las condiciones básicas para que exista un proceso comunicativo participativo y mediador, en el sentido que da Martín Barbero a la mediación: el diálogo ético y humano entre los actores sociales. Según este teórico (2012), para poder pensar la comunicación, disciplina que por su naturaleza evoluciona y cambia constantemente, había que salir de la zona de confort que las teorías tradicionales aportaban y "escuchar" y "ver" cómo la gente vive la comunicación y cómo la gente se comunica. Hay que entender que, en la comunicación, la realidad concreta supera los paradigmas teóricos funcionalistas y positivistas, concentrados en su mayoría en la información más que en la comunicación.

En respuesta a estos procesos de intercambio y cambio social, hay que pensar la comunicación desde las "matrices de identidad y los conflictos que articula la cultura" (Martín-Barbero, 2012, p.80), y por tanto formar un comunicador que motive y genere un diálogo intercultural. Lejos queda pensar que la misión de las facultades y escuelas de comunicación se limita a formar solamente periodistas y comunicadores organizacionales, más aun cuando estos

\footnotetext{
${ }^{1}$ Acrónimo de Director de Comunicación.
}

últimos durante las últimas tres décadas han sido limitados por la tautología de su propia denominación a comunicadores vinculados solamente con el ámbito empresarial, pues erróneamente se ha reducido y limitado el término organización a empresa, y la función máxima del comunicador a la de Dircom?.

El DirCom no es solo una nueva figura ubicada en el organigrama. Es el resultado de un proceso que surge en las empresas europeas a partir de nuevas necesidades propias que van de la mejora de la producción y la calidad a la optimalización de la competitividad, y de las relaciones laborales a la construcción de la imagen pública y la reputación... La inteligencia del DirCom consiste en asumir la triple naturaleza de la gestión empresarial, que considera que su estrategia y su gestión son por una parte, dirección, es decir, iniciativa e integración. Y por otra parte, administración, o sea, tratamiento de un sistema de relaciones sociales dentro y fuera de la organización. (Costa, 2011, p.101)

La función de este comunicador se limita desde su propia definición a pensar la comunicación desde la tecnología de la información, y, por tanto, "los comunicadores que no practican los fundamentos del diálogo pertenecen a 
la categoría de informadores" (Gamucio, 2004, p. 19). Desde la dialéctica de la comunicación y más específicamente desde el modelo de la 'Mediación Dialéctica de la Comunicación' (Piñuel, 1997), las interacciones comunicativas pueden ser estudiadas como fenómenos cuyos componentes (emisor, mensaje, receptor, código y canal) intervienen facilitando el intercambio social, requerido por la organización, y la interacción interpersonal, requerida por la necesidad de adaptación al entorno; entonces, aun las organizaciones tipo empresa y las organizaciones más complejas como las comunitarias, no pueden ser restringidas a una gestión desde la información con una lógica vertical, sino que deben ser estudiadas e intervenidas desde una visión estratégica con base en un proceso de diálogo constructivo subordinado a la cultura.

Como resultado, la formación de un nuevo comunicador implica "pensar los procesos de comunicación no desde las disciplinas, sino desde los problemas y las operaciones del intercambio social esto es desde las matrices de identidad y los conflictos que articula la cultura" (Martín-Barbero, 2012, p.80). Frente a este reto, las carreras de comunicación deben pensar sus propuesta formativas desde las necesidades de desarrollo propias del contexto al que responden. Así, nace la propuesta de un comunicador para el cambio social.

La comunicación para el cambio social es una comunicación ética, es decir, de la identidad y de la afirmación de valores; amplifica las voces ocultas o negadas, y busca potenciar su presencia en la esfera pública. Recupera el diálogo y la participación como ejes centrales; ambos elementos existían entrelazados con otros modelos y paradigmas y estaban presentes en la teoría como en un gran número de experiencias concretas. (Gamucio-Dragon, 2004, pp. 6-7)

\section{CONCLUSIÓN}

Se debe partir entonces de la premisa que la comunicación es un proceso social de producción de sentidos simbólicos; para poder hacer comunicación, primero se debe legitimarla en su mis- mo origen: la comunidad y su cultura. Entiéndase comunidad en su concepto más amplio. Así, siendo el Ecuador un país tan diverso y lleno de manifestaciones culturales tan particulares, no pode- 
mos pensar en modelos de comunicación, estrategias y mensajes producto de procesos de planificación exógenos. Por el contrario, "las comunidades deben ser agentes de su propio cambio y gestoras de su propia comunicación" (Gamucio-Dragon, 2011, p.37).

Así, pues, el éxito de las estrategias de comunicación se define por la capacidad de empoderamiento y autorreconocimiento de las prácticas culturales de sus propios actores, quienes se transforman en los responsables y ejecutores de sus propios mensajes y de sus propios instrumentos. Este autorreconocimiento demanda que la planificación de la comunicación rompa con el paradigma que el medio es el mensaje, y parta de la comprensión que son los procesos y no los instrumentos lo que promueve el diálogo participativo, la negociación de sentidos y por tanto la comunicación. Tal es el caso particular de la tecnología, que, dentro de la propuesta de la comunicación para el cambio social, plantea que

"el uso de la tecnología debe dimensionarse de acuerdo con las necesidades de cada proceso comunicacional. La capacidad de apropiación que desarrollen los actores involucrados define, en cada etapa del proceso, las características de la tecnología que debe usarse". (Gamucio-Dragon, 2011, p.38) 


\section{REFERENCIAS:}

Aparicio, R. (2014) Comunicación ambiental: aproximaciones conceptuales para un campo emergente, en Comunicación y sociedad n25, junio de 2016. Recuperado de http://www.scielo.org.mx/ scielo.php?script=sci_arttext\&pi$d=$ S0188-252X2016000100009

Bañuelos, J. (2013) Claves de la fotografía digital contemporánea: Prácticas, competencias, socialización y tendencias, en Islas, O y Ricaurte, P. (coord.), Investigar las redes sociales. Recuperado de http:// www.razonypalabra.org.mx/Libro_IRS/01_Banuelos_IRS.pdf

Costa, J. (2011). El ADN del Dircom: origen, necesidad, expansión y futuro de la dirección de comunicación. Barcelona: CPC.

Gumucio-Dragon, A. (2004, Agosto). E cuarto mosquetero- la comunicación para el cambio social. Revista de Investigación \& Desarrollo. 12, 2-23.

Gumucio-Dragon, A. (2011). Comunicación para el cambio social: clave del desarrollo participativo. En J. M. Pereira \& A. Cadavid (Eds.), Comunicación, desarrollo y cambio social. Interrelaciones entre comunicación, movimientos ciudadanos y medios (pp. 19 - 35). Bogotá: Pontificia Universidad Javeriana. Martín-Barbero, J. (2012). De la Comunicación a la Cultura: perder el "objeto" para ganar el proceso. Signo y Pensamiento, XXX (60), 76-84. Recuperado de http://www.redalyc. org/articulo.oa?id=86023575006

Martín Serrano, M. (1977). La mediación Social. Madrid: Akal.

Molina, M. (2010) La crisis de los grandes periódicos. Infoamérica 2 (139148). Recuperado de http://www. infoamerica.org/icr/n02/martinez_molina.pdf

Morabes, P. (2013). La fundamentación teórica problemáticas emergentes y líneas de investigación en comunicación/ educación: algunas reflexiones iniciales ante la implementación de políticas públicas de inclusión educativas en Argentina. La Plata, Argentina: Universidad Nacional de la Plata.

Piñuel, J. (1997). Teoría de la Comunicación y gestión de las organizaciones. Madrid: Síntesis.

Ortiz, M. (2003). México: Profesiones emergentes para los comunicadores, Chasqui revista Latinoamericana de Comunicación, No 84, (Ciespal), diciembre de 2003, 
recuperado de http://chasqui. ciespal.org/index.php/chasqui/ article/view/1502/1530

Ortiz, Q. (1998). De los medios a las mediaciones o las preguntas por el sentido. Iconos, No4, Flacso.

Rodríguez Santibáñez, I. (2017). Adiós a la prensa escrita, en Forbes, 12 de agosto de 2013. Recuperado de http://www.forbes.com.mx/
adios-a-la-prensa-escrita/\#gs.vSODZQO

Torres Nabel, L. ( 2013 ) Cómo analizar redes sociales en Internet. El caso Twitter en México, en Islas, O y Ricaurte, P. (coord.), Investigar las redes sociales. Recuperado de http:// www.razonypalabra.org.mx/Libro_IRS/01_Banuelos_IRS.pdf 
